\title{
Unusual presentation of Precursor B Lymphoblastic Lymphoma originating from the stomach: One more case and review of the literature
}

\author{
Maria G I oannou ${ }^{1}$, Eleni Bouronikou ${ }^{2}$, Spiros Potamianos ${ }^{3}$, Evangelos Kouvaras ${ }^{1}$, Stavros \\ Pateras $^{1}$, Maria Samara ${ }^{1}$, George K Koukoulis ${ }^{1}$
}

1. Department of Pathology, School of Medicine, University of Thessaly, Larissa, Greece. 2. Division of Hematology, University Hospital of Larisa, Greece. 3. Department of Gastroenterology, School of Medicine, University of Thessaly, Larissa, Greece.

Correspondence: Maria G Ioannou. Address: Department of Pathology, University of Thessaly, School of Medicine, Biopolis, Larissa 41110, Greece. Email: mioan@med.uth.gr

Received: December 17, 2013 Accepted: January 24, 2014

DOI : $10.5430 /$ jst.v4n1p56

URL: http://dx.doi.org/10.5430/jst.v4n1p56

Online Published: February 6, 2014

\section{Abstract}

Precursor B lymphoblastic lymphoma (PBL) is an aggressive neoplasm of precursor lymphocytes of B-cell lineage. Extranodal sites are often affected, however gastrointestinal involvement is rarely encountered. We report a case of PBL diagnosed in gastric biopsy of a 72-year-old female. Endoscopy showed multiple polypoid lesions in the stomach. Histologically, the tumor cells exhibited blastic morphology and immunohistochemical expression of terminal deoxynucleotidyl transferase (TdT), CD79a, CD20, CD10 and PAX-5, whereas they were CD34, MPO, and CD3 negative. The diagnosis of this rare entity in unusual extranodal sites such as the stomach is challenging, and it is based on detailed histopathological and immunohistochemical analysis combined to clinical correlations.

\section{Key words}

B lymphoblastic lymphoma, Stomach, Endoscopy, Pathology

\section{Introduction}

B Lymphoblastic leukemias and B lymphoblastic lymphomas represent the spectrum of clinical presentations of the same neoplasm of precursor B cells or lymphoblasts. Based on morphologic, genetic, and immunophenotypic features the World Health Organization (WHO) classification has unified these neoplasms as precursor B-cell lymphoblastic leukemia/ lymphoma ${ }^{[1]}$. Precursor B-cell lymphoblastic lymphoma (PBL) occurs more frequently in children and adolescents with a male predominance. Although it primarily involves lymph nodes and various extranodal sites ${ }^{[1-3]}$, however primary gastric involvement has been reported extremely rarely. In the present study, we describe a case of PBL diagnosed in the stomach, showing the typical morphological and immunohistochemical profile of the tumor. We also review the relevant literature and summarize the previously reported clinical, endoscopical and histopathological features of the disease. 


\section{Case presentation}

A 72-year-old female presented to the University Hospital of Thessaly with fatigue, nausea and vomiting for the last two months. The patient had no previous medical history. On clinical examination there was no evidence of peripheral lymphadenopathy or hepatosplenomegaly. Laboratory investigation revealed normochromic normocytic anaemia. Computed tomography (CT) scans of the head and neck, chest, and abdomen, demonstarated many small hypovascular lesions in the stomach. Lymphadenopathy was not detected. Swelling of the left parotid gland was also noted.

Esophagogastroduodenoscopy identified multiple polypoid lesions in the stomach (Figure 1). Biopsy samples taken from the polyps were routinely processed in paraffin for light microscopy and immunohistochemistry. Parotid biopsy was not performed. Microscopic examination of hematoxylin and eosin stained sections showed diffuse infiltration of the gastric mucosa and submucosa by uniform, medium-sized immature lymphoid cells, with oval or convoluted nuclei, evenly dispersed nuclear chromatin and a high nuclear/cytoplasmic ratio. Mitotic figures were easily found (Figure 2a). Immunohistochemical analysis showed that the neoplastic cells were positive for CD20, TdT, CD10, CD79a, and PAX-5 (Figures 2b-e) whereas they were negative for CD34, myeloperoxidase, CD117 and CD3. About 90\% of the tumor cells were reactive for Ki-67. On the basis of morphology and immunophenotype, a diagnosis of gastric precursor PBL was assigned. There was no evidence of precursor B lymphoblasts in the peripheral blood and bone marrow trephine showed minimal involvement represented $5 \%$ of the cellularity.
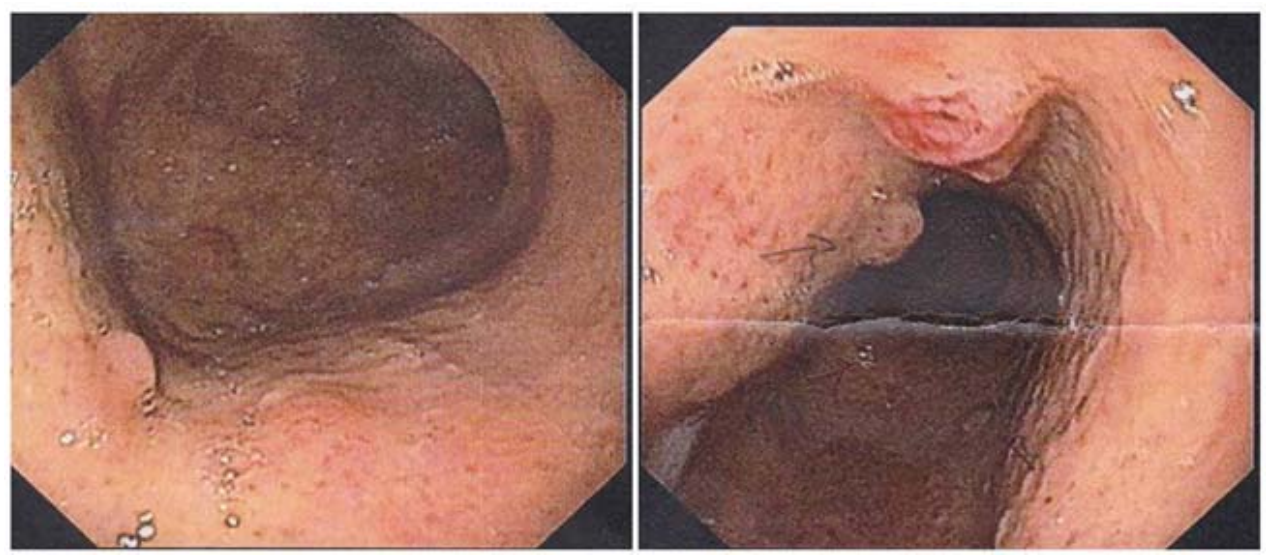

Figure 1. Endoscopy revealed multiple polypoid lesions

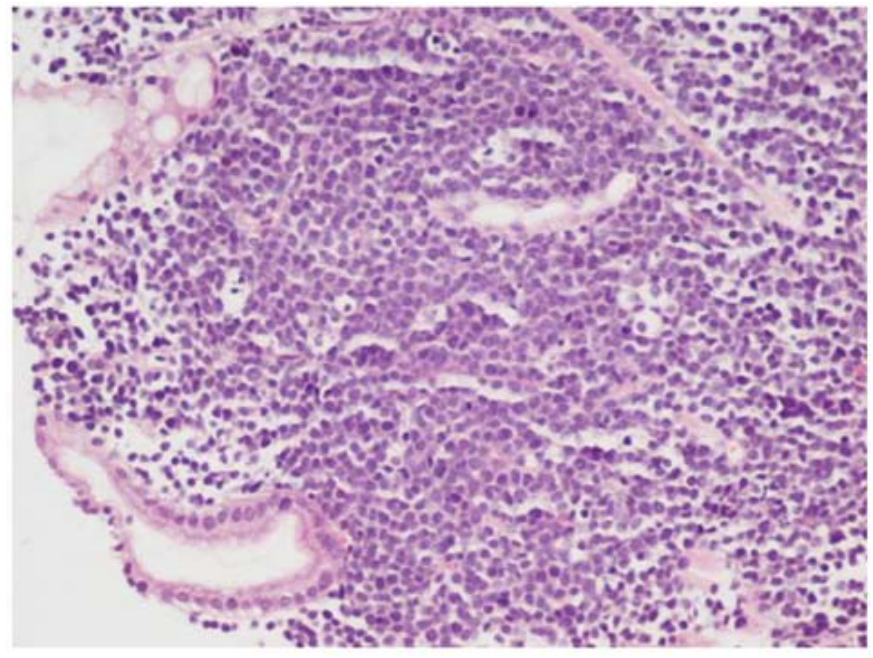

Figure 2a. Histopathology shows a monotonous infiltration of medium-sized lymphoid cells with a high nuclear-cytoplasmic ratio. Hematoxylin and Eosin staining, original magnification X20. 
The patient received one cycle of chemotherapy (CHOP plus Rituximab), which was followed by severe, grade four neutropenia and thrombocytopenia. Ten days after the initial treatment, she presented with subdural hematomas and a few days later, the disease was further complicated by severe lung infection caused by Klebsiella pneumonia. The patient finally died of concurrent acute respiratory distress syndrome (ARDS) and sepsis.
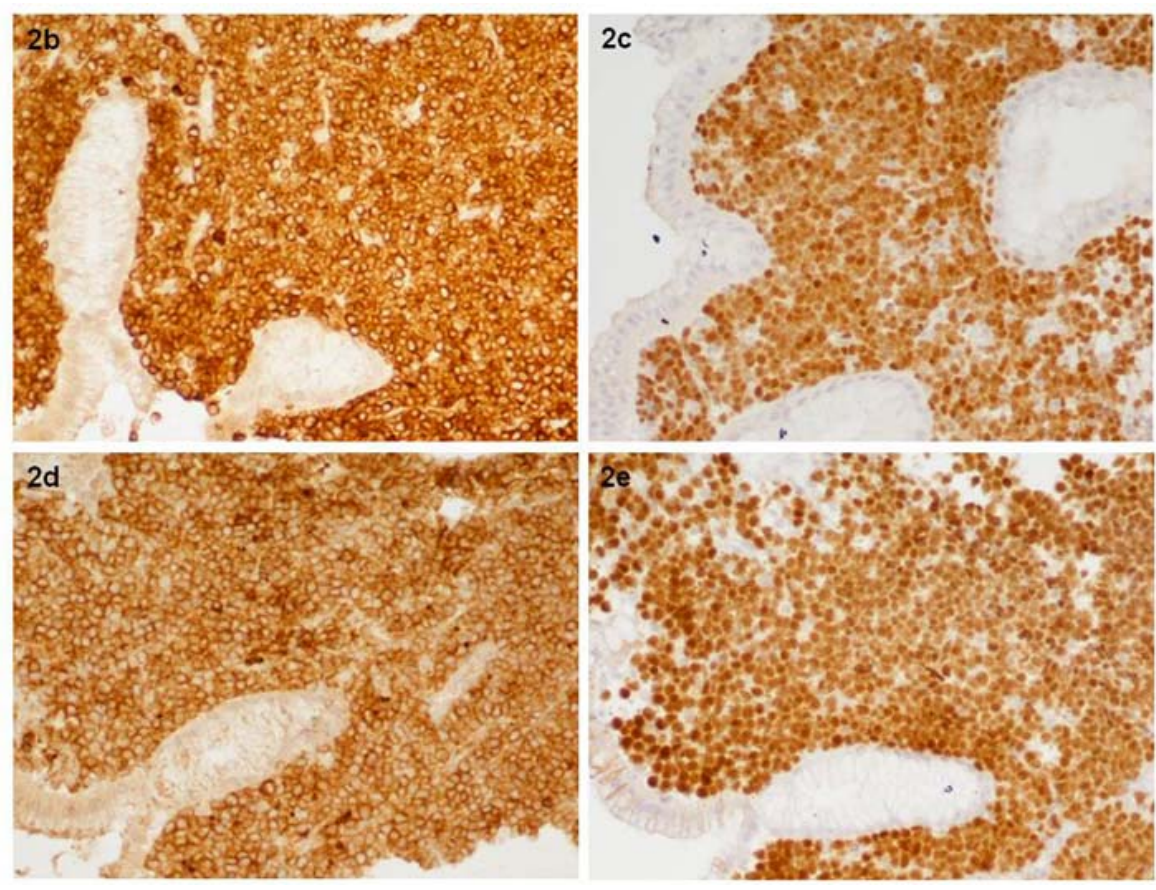

Figure2b, 2c, 2d, 2e. The lymphoblasts express CD20 (2b), TdT (2c), CD10 (2d) and PAX-5 (2e) Immunohistochemical staining, original magnificationX20

\section{Discussion}

Precursor B lymphoblastic lymphoma (PBL) is a neoplasm of lymphoblasts committed to the B-cell lineage ${ }^{[1]}$. Pathological and immunohistochemical features of PBL are indistinguishable from those of precursor B lymphoblastic leukemia and these tumors are considered to represent different clinical manifestations of the same tumor. By convention, the term lymphoma is used as the process is confined to a mass lesion with no or minimal evidence of peripheral blood and bone marrow. With extensive bone marrow involvement consisting of more than $25 \%$ of the cellularity and peripheral blood involvement, the term lymphoblastic "leukemia" is appropriate.

PBL is uncommon in adults. Most cases reported are less than 18 years old with a male predominance. Patients with PBL without leukemia are usually asymptomatic, and most of them have limited stage disease. Marrow and peripheral blood involvement may be present but the percentage of lymphoblasts in the bone marrow is less than $25 \%$. The most frequent sites of involvement in PBL, are the skin, soft tissue, bone and lymph nodes. Head and neck presentations are particularly common in children ${ }^{[1]}$. Involvement of the stomach has been described extremely rarely, in case reports or in case studies, the latter mainly before the advent of immunohistochemistry ${ }^{[3-8]}$. All the previously reported primary gastric PBLs as well as our case have occurred in adults ${ }^{[3,4,9]}$.

The endoscopical features of primary gastric lymphomas show marked variation. Ulcerated, polypoid, granular and nodular or infiltrative lesions have been described. According to previous publications, heterogenity in the macroscopical features of primary gastric PBL has also been noted. A case with diffusely thickened gastric wall and large ulcerative tumor has been described as well as another case showing multiple flat elevated lesions with irregular mucosa ${ }^{[4,9]}$. The 
endoscopical features in our patient were those of multiple polypoid lesions. A summary of the clinical, and endoscopical data of the previously published cases is provided in Table 1.

Table 1. Summary of clinical data of the reported cases of primary gastric PBL

\begin{tabular}{|c|c|c|c|c|}
\hline Reference & Age (yo) & Sex & Primary localization & Macroscopic features \\
\hline Iwamuro et al $2013^{[9]}$ & 85 & $\mathrm{~F}$ & Stomach & Multiple elevated mucosal lesions \\
\hline He at al $2008^{[4]}$ & 37 & $\mathrm{~F}$ & Stomach & Neoplastic ulcer \\
\hline Lin et al $2000^{[3]}$ & 25 & M & Stomach, colon & Friable gastric mass \\
\hline Our patient 2014 & 72 & $\mathrm{~F}$ & Stomach & Polypoid lesions \\
\hline
\end{tabular}

Table 2. Summary of immunophenotyping profile and molecular data of the reported cases of primary gastric PBL

\begin{tabular}{|c|c|c|c|c|c|c|c|c|c|c|c|c|c|}
\hline Reference & TdT & CD20 & CD79a & CD10 & CD34 & CD45 & PAX5 & CD3 & CD5 & CD99 & CD43 & CD45 RB & $\begin{array}{c}\text { Molecular } \\
\text { Data }\end{array}$ \\
\hline $\begin{array}{l}\text { Iwamuro et } \\
\text { al } 2013^{[9]}\end{array}$ & + & + & N/A & + & N/A & N/A & N/A & - & N/A & N/A & N/A & N/A & N/A \\
\hline $\begin{array}{l}\text { He et al } \\
2008^{[4]}\end{array}$ & + & - & + & + & N/A & N/A & N/A & N/A & N/A & + & N/A & N/A & $\begin{array}{l}\text { IGH gene } \\
\text { rearrangement }\end{array}$ \\
\hline $\begin{array}{l}\text { Lin et al } \\
2000^{[3]}\end{array}$ & + & - & + & + & - & N/A & N/A & N/A & N/A & N/A & + & + & $\begin{array}{c}I G H \text { gene } \\
\text { rearrangement }\end{array}$ \\
\hline $\begin{array}{l}\text { Our patient } \\
2014\end{array}$ & + & + & + & + & - & N/A & + & - & N/A & N/A & N/A & N/A & N/A \\
\hline
\end{tabular}

+Positive, - negative, NA: not available or not done, IGH: Immunoglobulin heavy chain

The histopathological differential diagnosis of PBL includes precursor T-lymphoblastic lymphoma, acute myeloid leukemia-myeloid sarcoma, and Burkitt's lymphoma. Microscopically, the lymphoblasts are small to medium sized with fine dispersed chromatin, indinstinct nucleoli, and a high mitotic rate ${ }^{[10]}$. Immunohistochemically, B lymphoblasts are positive for TdT which is never seen in Burkitt's lymphoma and almost always express the B-cell antigens CD19 and CD79a ${ }^{[1]}$. The T-cell associated antigen CD43 is usually positive in PBL, but expression of other T-cell antigens is negative. CD34 could be positive, however immunohistochemical detection of this antigen is much less sensitive than flow cytometry. In addition, B lymphoblasts never express myeloperoxidase which aids in differential diagnosis with acute myeloid leukaemia. Lymphoblasts are also positive for CD10, whereas they show variable expression of CD20 and CD22. Nearly all cases of B lymphoblastic leukemia have clonal DJ rearrangements of the immunoglobulin heavy chain gene (IGH gene rearrangements). Two of the previously reported primary gastric PBL cases have been studied by PCR and showed rearrangements of the IGH gene. These cases did not express CD20 and the molecular studies were used to support the histopathological diagnosis. In this case, the monomorphic proliferation of lymphoid cells showing expression of TdT and of B-cell markers such as PAX-5, CD20, CD79a together with the absence of MPO, CD34, CD117, and CD3 immunoexpression, led to a final diagnosis of precursor B lymphoblastic lymphoma. A diagnosis of B lymphoblastic leukaemia was excluded because the patient had a mass confined in the stomach, without peripheral blood involvement whereas the marrow involvement was limited and represented only $5 \%$ of the overall cellularity. In our case, we did not apply molecular studies, as the diagnosis was straightforward. A summary of the immunohistochemical and molecular data of the previously published cases is provided in Table 2 .

The prognosis of PBL is considered relatively favourable, especially in children ${ }^{[11]}$. An aggressive therapy following the correct histopathological diagnosis, is very important ${ }^{[12-14]}$. The treatment includes chemotherapy combined with monoclonal anti CD20 (Rituximab) and radiotherapy in selected cases. Our patient received CHOP plus Rituximab, however she developed subdural hematomas and she died few weeks after the initial diagnosis. Iwamuro et al reported a patient with primary gastric PBL who received THP-COP chemotherapy (tetrahydropyranyl adriamycin, cyclophosphamide, vincristine, and prednisolone) in combination with Rituximab. Moreover, radiotherapy was performed for palliation of her pain. Nevertheless, the lymphoma lesions did not respond to the treatment and the patient died after nine months ${ }^{[9]}$. Another patient was initially treated with six cycles of CHOP (cyclophosphamide, doxorubicin, vincristine, and 
prednisone $)^{[3]}$. He had a relapse treated with six cycles of CHOP and additionally with five cycles of H-CVAD for residual disease. Biopsy specimens were negative at the end of therapy however, bone marrow infiltration was detected one year after the diagnosis. Surgical resection followed by chemotherapy has been reported in the third case. The patient was in complete remission six months after the diagnosis ${ }^{[4]}$.

In conclusion, despite the very low incidence of gastric PBL, hematologists, gastroentrologists, surgeons, and histopathologists should be alert to the possibility because, it is a potentially curable disease. The histopathological diagnosis and clinical differential diagnosis of PBL requires detailed immunohistochemical analysis and an experienced pathologist as well as careful clinicopathological correlation.

\section{Competing interests}

The authors declare that they have no competing interests.

\section{References}

[1] Borowitz MJ, Chan JKC. Precursor lymphoid neoplasms. World Health Organization Classification of Tumours of Haematopoietic and Lymphoid Tissues. Lyon, France: IARC Press; 2008: 167-178.

[2] Soslow RA, Baergen RN, Warnke RA. B-lineage lymphoblastic lymphoma is a clinicopathologic entity distinct from other histologically similar aggressive lymphomas with blastic morphology. Cancer. 1999 Jun 15; 85(12): 2648-54. http://dx.doi.org/10.1002/(SICI)1097-0142(19990615)85:12<2648::AID-CNCR22>3.0.CO;2-R

[3] Lin P, Jones D, Dorfman DM, Medeiros LJ. Precursor B-cell lymphoblastic lymphoma: a predominantly extra-nodal tumor with low propensity for leukemic involvement. Am J Surg Pathol. 2000 Nov; 24(11): 1480-90. http://dx.doi.org/10.1097/00000478-200011000-00003

[4] He MX, Zhu MH, Liu WQ, Wu LL, Zhu XZ. Primary lymphoblastic B-cell lymphoma of the stomach: a case report. World Journal of Gastroenterology. 2008 May 21; 14(19): 3101-4. http://dx.doi.org/10.3748/wjg.14.3101

[5] Lee JD, Ko YH, Park JH, Min KR, Park KN. Histological and immunohistochemical studies on primary gastrointestinal lymphomas. Journal of Korean medical science. 1993 Feb; 8(1): 17-23.

[6] Takahashi H and Hansmann ML. Primary gastrointestinal lymphoma in childhood (up to 18 years of age): a morphological, immunohistochemical and clinical study. Journal of Cancer Research and Clinical Oncology. 1990; 116(2): 190-6. http://dx.doi.org/10.1007/BF01612676

[7] Mohri N. Primary gastric non-Hodgkin's lymphomas in Japan. Virchows Archiv. 1987; 411(5): 459-66. http://dx.doi.org/10.1007/BF00735227

[8] van Krieken JH, Otter R, Hermans J, van Groningen K, Spaander PJ, van de Sandt MM, Keuning JF, Kluin PM. Malignant lymphoma of the gastrointestinal tract and mesentery. A clinico-pathologic study of the significance of histologic classification. The American Journal of Pathology. 1989 Aug; 135(2): 281-9.

[9] Iwamuro M, Kawai Y, Yamawaki Y, Takata K, Yamamoto K. Precursor B Lymphoblastic Lymphoma Involving the Stomach. Case Rep Gastrointest Med. 2013;2013:930918. PMID:23840980 http://dx.doi.org/10.1155/2013/930918

[10] Knowles DM. Lymphoblastic lymphoma. Neoplastic Hematopathology. Baltimore, Md, USA: Williams \&Wilkins; 1992: 715-748.

[11] Maitra A, McKenna RW, Weinberg AG, Schneider NR, Kroft SH. Precurson B lymphoblastic lymphoma. A study of nine cases lacking blood and bone marrow involvement and review of the literature. Am J Clin Pathol. 2001; 115: 868-875.

[12] Zinzani PL, Bendandi M, Visani G, Gherlinzoni F, Frezza G, Merla E, Manfroi S, Gozzetti A, Tura S. Adult lymphoblastic lymphoma: clinical features and prognostic factors in 53 patients. Leuk Lymphoma. 1996 Nov; 23(5-6): 577-82. http://dx.doi.org/10.3109/10428199609054867

[13] Le Gouill S, Lepretre S, Briere J, Morel P, Bouabdallah R, Raffoux E, Sebban C, Lepage E, Brice P. Adult lymphoblastic lymphoma: a retrospective analysis of 92 patients under 61 years included in the LNH87/93 trials. Leukemia. 2003 Nov; 17(11): 2220-4. http://dx.doi.org/10.1038/sj.leu.2403095

[14] Kantarjian HM, O’Brien S, Smith TL, Cortes J, Giles FJ, Beran M, Pierce S, Huh Y, Andreeff M, Koller C, Ha CS, Keating MJ, Murphy S, Freireich EJ. Results of treatment with hyper- CVAD, a dose-intensive regimen, in adult acute lymphocytic leukemia. J Clin Oncol. 2000 Feb; 18(3): 547-61. 